\title{
Spanning Trees with generalized degree constraints arising in the design of wireless networks
}

\author{
Luís Gouveia, Pedro Moura, Amaro de Sousa
}

\begin{abstract}
In this paper we describe a minimum spanning tree problem with generalized degree constraints which arises in the design of wireless networks. The signal strength on the receiver side of a wireless link decreases with the distance between transmitter and receiver. In order to work properly, the interference on the receiving part of the link must be under a given threshold. In order to guarantee this constraint, for each node we impose a degree constraint that depends on the "length" of the links adjacent to the corresponding node, more precisely, nodes adjacent to long links must have a smaller degree and vice-versa. The problem is complicated by considering different signal strengths for each link. Increasing the strength in a link increases the cost of the link. However, it also reduces the maximum allowed degree on its end nodes. We create two models using adequate sets of variables, one may be considered an extended version of the other, and relate, from a theoretical perspective, the corresponding linear programming relaxations.
\end{abstract}

\section{Introduction}

In this paper we consider a wireless network design problem that generalizes a problem previously defined and studied in [4] (see also [2, 3]). These problems also generalize the well-known degree constrained spanning tree problem (see [1] and

Luís Gouveia

CIO-DEIO, Bloco C6-Piso 4, Faculdade de Ciências da Universidade de Lisboa, Cidade Universitária, Campo Grande, 1749-016 Lisboa, Portugal, e-mail: legouveia@ fc.ul.pt

Pedro Moura

CIO-DEIO, Bloco C6-Piso 4, Faculdade de Ciências da Universidade de Lisboa, Cidade Universitária, Campo Grande, 1749-016 Lisboa, Portugal, e-mail: pmmoura@fc.ul.pt

Amaro de Sousa

Instituto de Telecomunicações, Universidade de Aveiro, 3810-193 Aveiro, Portugal, e-mail: asou@ua.pt 
the references inside) in the sense that they consider node degree dependent costs and more complicated degree constraints (the constraint on the degree of a node depends on the edges adjacent to it in the solution). Section 2 describes and motivates the new problem. Section 3 describes several models for the problem.

\section{Description and motivation of the problem}

In point-to-point wireless networks, each network connection is implemented through a point-to-point wireless transmission system (wireless link, for short) composed by a pair of transmitter/receiver antennas and signal processing units (one at each side of the connection) working on a frequency channel, chosen from a possible set of channels. Thus, consider an undirected graph $G=(V, E)$ where $V=\{1, \ldots, n\}$ is the set of network nodes and $E \subseteq V^{2}$ is the set of edges $\{i, j\}$, representing each network connection. A network node with wireless links for different neighbour nodes must use different frequency channels. In most wireless technologies, due to the scarcity of the spectrum, there is a limited set of available frequency channels and many of them are partially overlapped between each other. Therefore, in a node using partially overlapped channels to different neighbour nodes, part of the transmitted signal on one channel is added as interference to the received signal on the other channel. Note that the signal strength on the receiver side of a wireless link decreases with the distance between transmitter and receiver antennas due to attenuation and other propagation effects. In order to work properly, the received signal must be such that the signal-to-interference-and-noise ratio (SINR) on the receiver is above a required threshold. Therefore, the coverage of a wireless link, i.e., the maximum distance between antennas that make the link work properly, depends on the amount of interference introduced by the other frequency channels on its end nodes. When a given wireless link cannot meet the required SINR threshold, we can consider three possible cases.

Case 1 Several costs / A single maximum degree parameter: In this case, we assume that pairs of nodes with higher distance have more expensive wireless links, with a higher power transmission, in order to raise the SINR over the required threshold. A parameter $D$ is set as the maximum degree for each node (based on the available frequency channels) and, for each pair of nodes $i$ and $j$ with a distance equal to $d_{i j}$, a cost value $c_{\{i, j\}}$, which depends on $d_{i j}$, is defined as the least cost wireless link that can still provide the required SINR whatever the degree of its end nodes is. This is the case adopted in [4] where 3 types of wireless links were considered, each one with a different coverage and cost.

Case 2 A single cost / Several maximum degree parameters: In this case, we assume that there is only one type of wireless link with an associated cost value $c$ and such that it is not used when the required SINR threshold is not met. For each pair of nodes $i$ and $j$ with a distance equal to $d_{i j}$, a degree parameter $D_{\{i, j\}}$, which depends on $d_{i j}$, is defined as the maximum de- 
gree of both $i$ and $j$ such that interference does not jeopardize the required SINR threshold for the wireless link to work properly.

Case 3 Several costs / Several maximum degree parameters. In this case, we assume that there are $T$ types of wireless links with associated (increasing) costs $f_{t}, 1 \leq t \leq T$, and the degree of its endnodes depends on the type of link installed. Consider a pair of nodes $i$ and $j$ with a distance equal to $d_{i j}$. We define $D_{\{i, j\}}^{t}$, which depends on $d_{i j}$, as the maximum degree on both nodes $i$ and $j$, if we install a wireless link of type $t$ between these two nodes. Then, we can install a higher cost wireless link, allowing both nodes to have higher degrees, or install a lower cost wireless link constraining the degrees of nodes $i$ and $j$ to be lower. That is, we have a cost model for a wireless link to be installed between two nodes, $i$ and $j$, which not only depends on the distance between those two nodes, but also depends on the degree that nodes $i$ and $j$ will have in the solution of the problem. Then, for each pair of nodes $i$ and $j$ we define a cost $c_{\{i, j\}}^{m}$, which gives the cost of the cheapest cost wireless link that can be used, assuming that $m$ is the maximum of the degrees of nodes $i$ and $j$.

Note that cases 1 and 2 are particular cases of case 3. In fact, if we consider just one type of wireless link we obtain case 2. Also, case 1 is a particular case of case 3 when we assume that all types of wireless links allow the degree of its end nodes to be the maximum degree $D$ i.e., the degree parameters $D_{\{i, j\}}^{t}$ are equal to $D$ for all pairs of nodes $i$ and $j$ and all types $t$ of wireless links. In the next section we describe several models for this more general case 3 .

\section{Formulations}

In this section we describe two integer linear formulations for the problem. Consider binary variables $x_{\{i, j\}}$ indicating whether edge $\{i, j\} \in E$ is selected, as well as binary variables $y_{i}^{d}$ indicating whether node $i \in V$ has degree equal to $d \in\{1, \ldots, D\}$ in the solution. These variables were used in the models introduced in the works $[2,3,4]$ where problems with non linear costs associated to the node degrees were studied. The two models studied in this paper use the previous two sets of variables. They differ, however, on the set of variables that characterize the type of links to be installed.

\section{$3.1 \mathrm{Model}\left(P_{1}\right)$}

Besides the two sets of variables $x$ and $y$, model $\left(P_{1}\right)$ also uses binary variables $v_{\{i, j\}}^{m}$ indicating whether the edge $\{i, j\} \in E$ is selected and the maximum degree between nodes $i$ and $j$ is $m$ (with $m=2, \ldots, D$ ). Clearly, these variables are not defined for 
$m=1$, since we cannot have an edge where the degree of both endpoints is equal to 1. The problem can then be formulated as $\left(P_{1}\right)$ (we denote by $E(i) \subseteq E$ the set of edges incident on node $i$ ). The objective cost function is straightforward.

$$
\begin{aligned}
& \left(P_{1}\right) \min \sum_{\{i, j\} \in E} \sum_{m=2}^{D} c_{\{i, j\}}^{m} \cdot v_{\{i, j\}}^{m} \\
& \text { s.to: }\left\{\{i, j\} \in E: x_{\{i, j\}}=1\right\} \text { is a SpTree } \\
& \sum_{d=1}^{D} d \cdot y_{i}^{d}=\sum_{\{i, j\} \in E(i)} x_{\{i, j\}} \quad i \in V \\
& \sum_{d=1}^{D} y_{i}^{d}=1 \quad i \in V \\
& x_{\{i, j\}}=\sum_{m=2}^{D} v_{\{i, j\}}^{m} \quad\{i, j\} \in E \\
& v_{\{i, j\}}^{m} \leq y_{i}^{m}+y_{j}^{m} \quad\{i, j\} \in E ; m=2, \ldots, D \\
& 2 \cdot v_{\{i, j\}}^{m} \leq \sum_{d=1}^{m}\left(y_{i}^{d}+y_{j}^{d}\right) \quad\{i, j\} \in E ; m=2, \ldots, D-1 \\
& x_{\{i, j\}} \in\{0,1\} \quad\{i, j\} \in E \\
& v_{\{i, j\}}^{m} \in\{0,1\} \\
& \{i, j\} \in E ; m=2, \ldots, D \\
& y_{i}^{d} \in\{0,1\} \quad i \in V ; d=1, \ldots, D
\end{aligned}
$$

Constraints (2), stating that the solution is a Spanning Tree, are given in a generic form and can be written in several ways (see [5]). Constraints (3) and (4) define the degree variables $y_{i}^{d}$ and guarantee that $y_{i}^{d}=1$ iff the number of edges adjacent to node $i$ is equal to $d$. Constraints (5) link the two sets of edge variables, $x_{\{i, j\}}$ and $v_{\{i, j\}}^{m}$, stating that, if edge $\{i, j\}$ is selected, then the maximum between the degrees of its endnodes must be a value in $\{2, \ldots, D\}$. Constraints (6) and (7) link the node variables $y_{i}^{d}$ with the edge variables $v_{\{i, j\}}^{m}$ : for a given edge $\{i, j\}$, constraints (6) guarantee that if $v_{\{i, j\}}^{m}=1$ then one of the nodes $i$ or $j$ must have a degree equal to $m$, and constraint (7) guarantees that neither one of these nodes has a degree greater than $m$. Constraints (8)-(10) define the domain of the variables.

The variables $v_{\{i, j\}}^{m}$ are sufficient to describe the objective function of the problem since the extra index indicates the maximum degree of the endpoints associated to each edge. In the next subsection we create a model with edge variables having two extra indexes, associated to the degrees of each endpoint. We will show that these extra variables, although leading to a model with more variables, permit us to write a model with fewer constraints since it is easier (we need fewer constraints) to relate the new variables with the degree variables $y_{i}^{d}$. Furthermore, with the new set of variables we can derive, hopefully strong, valid inequalities. 


\section{2 $\operatorname{Model}\left(P_{2}\right)$}

Besides the $x_{\{i, j\}}$ and $y_{i}^{d}$ variables, model $\left(P_{2}\right)$ also uses binary variables $z_{\{i, j\}}^{p q}$, indicating whether the edge $\{i, j\} \in E$ is selected and degree $(i)=p$ and degree $(j)=q$. Again, these variables are not defined for $(p, q)=(1,1)$. Before describing the new model, we note that the two sets of edge variables, $v_{\{i, j\}}^{m}$ and $z_{\{i, j\}}^{p q}$, can be related as follows

$$
\begin{aligned}
& v_{\{i, j\}}^{m}=\sum_{q=1}^{m} z_{\{i, j\}}^{m q}+\sum_{p=1}^{m-1} z_{\{i, j\}}^{p m} \quad\{i, j\} \in E ; m=2, \ldots, D \\
& \left(P_{2}\right) \min \sum_{\{i, j\} \in E} \sum_{m=2}^{D} c_{\{i, j\}}^{m} \cdot\left(\sum_{q=1}^{m} z_{\{i, j\}}^{m q}+\sum_{p=1}^{m-1} z_{\{i, j\}}^{p m}\right) \\
& \text { s.to: }\left\{\{i, j\} \in E: x_{\{i, j\}}=1\right\} \text { is a SpTree } \\
& \sum_{d=1}^{D} d \cdot y_{i}^{d}=\sum_{\{i, j\} \in E(i)} x_{\{i, j\}} \quad i \in V \\
& \sum_{d=1}^{D} y_{i}^{d}=1 \quad i \in V \\
& x_{\{i, j\}}=\sum_{p=1}^{D} \sum_{q=1}^{D} z_{\{i, j\}}^{p q} \quad\{i, j\} \in E \\
& p \cdot y_{i}^{p}=\sum_{\{i, j\} \in E(i)} \sum_{q=1}^{D} z_{\{i, j\}}^{p q} \quad i \in V ; p=1, \ldots, D \\
& x_{\{i, j\}} \in\{0,1\} \quad\{i, j\} \in E \\
& y_{i}^{d} \in\{0,1\} \quad i \in V ; d=1, \ldots, D \\
& z_{\{i, j\}}^{p q} \in\{0,1\} \quad\{i, j\} \in E ; p, q=1, \ldots, D
\end{aligned}
$$

The objective function follows straightforwardly from the objective function of the previous model and the linking constraints (11). Note the constraints (14) linking the degree variables with the new link variables, which are much easier to write in this model. These constraints state that, if the degree of node $i$ is $p$ then, in the solution, exactly $p$ edges are incident in that node, whatever the degree of node $j$ is (for $p=1$, the summation on $q$ starts at 2). Note that under (13), constraints (3) for a given node $i$, can be obtained by adding constraints (14) for all $p=1, \ldots, D$ and for the same $i$. Thus constraints (14) are a disaggregation of (3) and the latter can be omitted from the integer model. However, we will come back again to the weaker constraints (3) since we will see later that we can obtain a different valid model for the problem where we can use the weaker but more compact set (3) rather than the stronger but less compact set (14). For the moment, we also point out that there is no dominance relationship between the linear programming relaxations of the two models $\left(P_{1}\right)$ and $\left(P_{2}\right)$. 
As we have stated before, another advantage of using the new variables is that we can write, hopefully strong, valid inequalities such as,

$$
\sum_{q=1}^{D} z_{\{i, j\}}^{p q} \leq y_{i}^{p} \quad i \in V,\{i, j\} \in E(i) ; p=1, \ldots, D
$$

The valid inequalities (16) state that if edge $\{i, j\}$ is in the solution and node $i$ has degree equal to $p$, whatever the degree on node $j$ is, then the corresponding $y$ variable associated to node $i$ and degree $p$ must be equal to 1 . We do not need to consider inequalities (16) for $p=1$ because these are implied by constraints (14) for node $i$ and $p=1$.

Denoting by $\left(P_{2}^{*}\right)$ the model obtained by adding the inequalities (16) to model $\left(P_{2}\right)$ as well as the definitional constraints (11) (these do not improve the linear programming bound) we can prove the following result.

Proposition 1. The projection of the set of feasible solutions of the linear programming relaxation of $\left(P_{2}^{*}\right)$ on the subspace defined by the variables $x, y$ and $v$ is contained in the set of feasible solutions of the linear programming relaxation of the model $\left(P_{1}\right)$.

In the proof of Proposition 1 (not presented here), we did not make use of constraints (14) of model $\left(P_{2}^{*}\right)$. In fact, it is not difficult to see that, in the presence of the new constraints (16), we still obtain a valid model by using only the weaker constraints (3) instead of both (3) and (14) constraints. We denote by $\left(P_{2}^{* *}\right)$ this model. Our results show that this model is of interest only from a theoretical perspective, since what we gain by reducing in $n \cdot D$ the total number of constraints does not compensate the weakening of the corresponding linear programming relaxation.

Acknowledgements Sponsored by FCT, research grants POCTI-ISFL-1-152 and PTDC/EIA/64772/2006.

\section{References}

1. Cunha, A., Lucena,A.: Lower and upper bounds for the Degree-constrained Minimum Spanning Tree Problem. Networks 50(1), 55-66 (2007)

2. Duhamel, C., Gouveia, L., Moura, P., Souza, M.: Models and Heuristics for the $k$-degree constrained minimum spanning tree problem with node-degree costs. to appear in Networks (2011)

3. Gouveia, L., Moura, P.: Spanning Trees with Node Degree Dependent Costs and Knapsack Reformulations. Electronic Notes in Discrete Mathematics 36,985-992 (2010)

4. Gouveia, L., Moura, P., Sousa, A.: Prize collecting Steiner trees with node degree dependent costs. Computers \& Operations Research 38(1), 234-245 (2010)

5. Magnanti, T., Wolsey, L.: Optimal Trees. In: Ball, M.O., Magnanti, T.L., Monma, C.L., Nemhauser, G.L. (eds.) Handbooks in Operational Research and Management Science (1995)

6. Martin, R.K.: Using Separation Algorithms to Generate Mixed Integer Model Reformulations. Operations Research Letters 10(3), 119-128 (1991) 